\title{
La importancia de las publicaciones en español
}

\author{
The importance of Spanish language publications
}

\author{
Alberto Orozco Gutiérrez* \\ Citar como: Orozco GA. La importancia de las publicaciones en español. Acta Med Grupo \\ Angeles. 2021; 19 (2): 171. https://dx.doi.org/10.35366/100437
}

La literatura médica ha sido, de manera tradicional, un medio de difusión del conocimiento local entre médicos, desde 1812 que se inició la publicación del New England Journal of Medicine, y en 1864 que se inició la publicación de la Gaceta Médica de México, estas revistas surgieron como respuesta a la necesidad de que los médicos de la ciudad y localidades cercanas pudieran informarse mutuamente de los avances científicos, desde el principio constituyeron canales de comunicación adecuados y abiertos a la crítica de todo tipo.

La difusión del conocimiento debe ser el objetivo fundamental de las publicaciones científicas, de nada sirve que los grandes avances se encierren en espacios pequeños, deben alcanzar a la comunidad.

En la actualidad, el inglés se ha impuesto como la lengua científica por excelencia, el 98\% de las publicaciones científicas están escritas en inglés, incluyendo los trabajos de investigadores de países con otra lengua nativa. ${ }^{1}$ Esto genera una gran desigualdad en el acceso masivo a los conocimientos, en el mundo existen aproximadamente 480 millones de personas que hablan como lengua nativa español y pocos de ellos hablan inglés.

De acuerdo con el ranking EF EPI 2020 (Education first, English Proficiency Index), México tiene un nivel de aptitud muy bajo de conocimiento del inglés, ocupamos el lugar 82 de 100 países estudiados y el lugar 18 de 19 países latinoamericanos. $^{2}$ Esto origina que el 98\% de los conocimientos científicos no están al alcance de la población, y esto incluye a los médicos y trabajadores de la salud.
Es por ello por lo que el papel de las revistas en español es muy importante, nuestros autores y lectores deben ser nuestra principal preocupación, la difusión de conocimientos para nuestro medio es de trascendental importancia.

La medicina mexicana y latinoamericana tiene grandes avances y autores, debemos pugnar porque participen en la literatura en español, también debemos lograr que nuestros autores día a día sean mejores y que el consultar la literatura en español se vuelva una parte importante de la práctica médica, muchos lectores de lengua inglesa leen nuestros resúmenes y los consideran muy interesantes.

El tener alternativas lingüísticas en la literatura médica permite que el número de autores se multiplique, mejorando los canales de comunicación que promueven diversos conocimientos culturales y científicos; la medicina es una ciencia con muchos matices, la historia y la cultura de cada país son parte importante de ella.

Menospreciar los artículos en otras lenguas es menospreciar la propia, nuestras raíces y por último a nosotros mismos.

\section{REFERENCIAS}

1. Ramírez-Castañeda V. Disadvantages in preparing and publishing scientific papers caused by the dominance of the English language in science: the case of Colombian researchers in biological sciences. PLoS ONE. 2020; 15 (9): e0238372. Available in: https://doi. org/10.1371/journal.pone.0238372.

2. EF EPI. Índice del EF english proficiency. Reporte 2020. Disponible en: https://www.ef.com.mx/epi/.

* Editor Responsable. Acta Médica Grupo Ángeles.

\section{Correspondencia:}

Alberto Orozco Gutiérrez

Correo electrónico:

editoractamedicagrupoangeles@gmail.com

www.medigraphic.com/actamedica

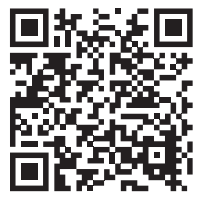

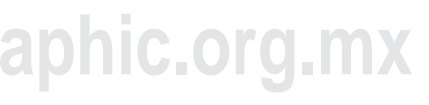

